\title{
Factors Influencing Lower Urinary Tract Symptoms in Advanced Cancer Patients With Chemotherapy-Induced Peripheral Neuropathy
}

\author{
Ok-Hee Cho ${ }^{1}$, Yang-Sook Yoo ${ }^{2}$, Joon Chul Kim ${ }^{3}$, Ran Hee Park ${ }^{4}$, Kyung-Hye Hwang ${ }^{5}$ \\ ${ }^{1}$ Department of Nursing, College of Nursing and Health, Kongju National University, Gongju, Korea \\ ${ }^{2}$ College of Nursing, The Catholic University of Korea, Seoul, Korea \\ ${ }^{3}$ Department of Urology, Bucheon St. Mary's Hospital, College of Medicine, The Catholic University of Korea, Bucheon, Korea \\ ${ }^{4}$ Department of Nursing, Seoul St. Mary's Hospital, Seoul, Korea \\ ${ }^{5}$ Department of Nursing, Suwon Science College, Hwaseong, Korea
}

Purpose: This study aimed to investigate the severity of lower urinary tract symptoms (LUTS) and to identify factors that influenced LUTS in advanced cancer patients with chemotherapy-induced peripheral neuropathy (CIPN).

Methods: This cross-sectional study included a total of 158 advanced cancer patients with CIPN. A structured questionnaire including the International Prostate Symptom Score and the Functional Assessment of Cancer Therapy/Gynecology Oncology Group/Neurotoxicity scale was used. Data were analyzed using the Pearson correlation coefficient and multiple regression analysis. Results: Nocturia was the most prevalent LUTS. A positive relationship was found between CIPN symptoms and LUTS. The duration of cancer diagnosis and the severity of CIPN were key factors that influenced LUTS.

Conclusions: The severity of CIPN symptoms was the most important predictor of LUTS. Nurses' care for advanced cancer patients should incorporate a comprehensive health assessment, which includes a history of treatment and physical neuropathic symptoms, for any patient complaining of CIPN symptoms.

Keywords: Neoplasm metastasis; Patients; Peripheral neuropathy; Lower urinary tract symptomsy

- Research Ethics: This study was approved by the Institutional Review Board of St. Mary's Hospital, The Catholic University of Korea (KCI13O-

ISI0345). Written consent was obtained from patients who agreed to participate in the study.

- Conflict of Interest: No potential conflict of interest relevant to this article was reported

\section{INTRODUCTION}

Peripheral neuropathy refers to sensory/motor neuropathy with pain, and it is a major complication that occurs in approximately $40 \%$ of cancer patients who receive chemotherapy [1]. Anticancer drugs such as vinca alkaloids, taxanes, and platinum agents are frequently associated with sensory neuropathic symptoms, such as tingling and numbness, as well as motor neuropathic symptoms that may be the cause of muscle weak- ness and some difficulty in walking [2,3]. Approximately $20 \%$ of cancer patients receiving concomitant chemotherapy complain of urinary retention or residual neuropathy $[4,5]$.

Lower urinary tract symptoms (LUTS) include dysuria and motor and sensory nerve dysfunction in the urethra. LUTS are divided into storage symptoms, such as frequency, nocturia, and urinary incontinence; voiding symptoms, such as hesitancy and straining; and post-voiding symptoms, such as residual urine sensation and postvoid dribbling [6]. A previous study
Corresponding author: Kyung-Hye Hwang (iD https://orcid.org/0000-0003-4571-1474 Department of Nursing, Suwon Science College, 288 Sejaro, Jeongnam-myun, Hwaseong 18516, Korea

E-mail: hkh@ssc.ac.kr / Tel: +82-31-350-2477 / Fax: +82-31-350-2076

Submitted: April 5, 2018 / Accepted after revision: July 12, 2018
This is an Open Access article distributed under the terms of the Creative Commons Attribution Non-Commercial License (http://creativecommons.org/licenses/by-nc/4.0/) which permits unrestricted non-commercial use, distribution, and reproduction in any medium, provided the original work is properly cited. 
reported that more than $70 \%$ of patients who received chemotherapy complained of LUTS, and LUTS may continue for months or even several years after chemotherapy [7,8]. LUTS have been associated with cancer treatment methods and the duration of disease after cancer diagnosis [8]. It has been reported that $68 \%$ of patients receiving chemotherapy complained of nocturia [9], and that $37.0 \%$ of cancer survivors showed urinary incontinence, but only $19.2 \%$ of them had received treatment [10].

Radiotherapy can irritate the bladder wall lining and muscle wall, leading to decreased bladder compliance and capacity [11]. Furthermore, chemotherapeutic drugs such as paclitaxel/ carboplatin therapy and cyclophosphamide can cause chemical-induced hemorrhagic cystitis symptoms (e.g., frequency, urgency, and pelvic pain) [12], which can increase the risk of cystitis, fibrosis, sphincter dysfunction, or bladder muscle stiffness $[11,12]$. As LUTS in advanced cancer patients can be a main reason for diminished quality of life (QoL), continuous management is necessary [7].

Limited studies have been conducted on LUTS-associated factors and QoL [8] and voiding dysfunction after cancer surgery [13]. This study aimed to investigate LUTS severity, to identify the factors that influenced LUTS in advanced cancer patients with chemotherapy-induced peripheral neuropathy (CIPN), and to provide baseline data for the development of nursing interventions aiming to improve micturition-related QoL.

\section{MATERIALS AND METHODS}

\section{Study Design}

This was a cross-sectional study designed to investigate the severity of LUTS and factors that influenced LUTS in advanced cancer patients with CIPN.

\section{Participants and Data Collection}

The participants in this study comprised 158 advanced cancer patients admitted to the Oncology Department in St. Mary's Hospital, The Catholic University of Korea, Seoul, South Korea. Participants agreed voluntarily to participate in this study after being informed about its purpose and procedures, and convenience sampling was used to recruit the sample of 158 participants.

The selection criteria included persons 18 years or older with stage III/IV gastric or lung cancer who had been treated with chemotherapy (>at least 1 cycle of platinum and/or taxane compound agents at the oncology clinic), had no preexisting
CIPN symptoms prior to initial chemotherapy, and were classified as National Cancer Institute-Common Toxicity Criteria (NCI-CTC) grade I or higher.

Data collection was conducted from February 2014 to June 2014, and the study received approval from the Institutional Review Board of St. Mary's Hospital, The Catholic University of Korea (KCI13OISI0345). Patients who met the selection criteria were informed about the purpose, methods, and procedures of the study, as well as about how to withdraw from participation. Written consent was obtained from patients who agreed to participate in the study. One researcher screened and selected grade I or higher patients based on the NCI-CTC grading system of peripheral neuropathy.

\section{Instruments}

Information about demographic and clinical characteristics was collected using self-reported measures and medical chart review. Peripheral neuropathy symptoms were measured using the neurotoxicity subscale of the Functional Assessment of Cancer Therapy/Gynecology Oncology Group/Neurotoxicity scale [14]. This tool comprised a total of 11 items pertaining to sensory, hearing, motor, and dysfunction symptoms. Each item was rated on a 5-point Likert scale (0-4), with a higher score indicating more severe symptoms.

LUTS were measured based on the International Prostate Symptom Score (IPSS) originally developed by Barry et al. [15] and translated into Korean by Choi et al. [16]. This tool consisted of 7 LUTS and IPSS-QoL measures associated with micturition. The severity of each LUTS level was rated on a 6-point scale (05 ), and the IPSS-QoL was rated on a 7-point scale (0-6). The severity of LUTS was classified into 3 grades: mild (0-7 points), moderate ( $8-19$ points), and severe (20-35 points). Thus, higher IPSS-QoL scores indicated lower micturition-related QoL.

\section{Statistical Analysis}

All collected data were analyzed using SAS ver. 9.2 (SAS Institute Inc., Cary, NC, USA). The general characteristics of the participants and LUTS severity were analyzed and presented as frequency, percentage, mean, and standard deviation. Differences in LUTS according to participants' general characteristics were analyzed through the t-test and analysis of variance. Correlations between CIPN symptoms and LUTS were analyzed using the Pearson correlation coefficient. Factors influencing LUTS were analyzed through multiple regression analysis. 


\section{RESULTS}

\section{Participants' General Characteristics}

The mean age of the participants was 61.3 years and $60.1 \%$ of them were male. Those with a body mass index of less than 23 $\mathrm{kg} / \mathrm{m}^{2}$ accounted for $53.2 \%$ of all participants, and those with an Eastern Cooperative Oncology Group (ECOG) score of 2 points accounted for $48.1 \%$. Additionally, $24.7 \%$ of the partici-

Table 1. Demographic characteristics of the participants $(n=158)$

\begin{tabular}{|c|c|c|c|c|}
\hline Characteristic & Total & LUTS & $\mathrm{t} / \mathrm{F}$ & P-value \\
\hline $\begin{array}{l}\text { Age }(\mathrm{yr}) \\
\quad<60 \\
\geq 60\end{array}$ & $\begin{array}{r}61.3 \pm 9.8 \\
67(42.4) \\
91(57.6)\end{array}$ & $\begin{array}{l}11.97 \pm 6.04 \\
15.75 \pm 7.21\end{array}$ & -3.48 & $<0.001$ \\
\hline $\begin{array}{l}\text { Sex } \\
\text { Male } \\
\text { Female }\end{array}$ & $\begin{array}{l}95(60.1) \\
63(39.9)\end{array}$ & $\begin{array}{l}13.34 \pm 6.46 \\
15.37 \pm 7.58\end{array}$ & -1.80 & 0.073 \\
\hline $\begin{array}{l}\text { Body mass index }\left(\mathrm{kg} / \mathrm{m}^{2}\right) \\
<23 \\
\geq 23\end{array}$ & $\begin{array}{l}84(53.2) \\
74(46.8)\end{array}$ & $\begin{array}{l}14.25 \pm 6.85 \\
14.03 \pm 7.16\end{array}$ & 0.20 & 0.842 \\
\hline $\begin{array}{c}\text { ECOG } \\
1 \\
2 \\
3\end{array}$ & $\begin{array}{l}51(32.3) \\
76(48.1) \\
31(19.6)\end{array}$ & $\begin{array}{l}11.55 \pm 5.72 \\
14.07 \pm 6.20 \\
18.61 \pm 8.47\end{array}$ & 11.19 & $<0.001$ \\
\hline $\begin{array}{l}\text { Diagnosis } \\
\text { Gastric cancer } \\
\text { Lung cancer }\end{array}$ & $\begin{array}{r}39(24.7) \\
119(75.3)\end{array}$ & $\begin{array}{l}10.92 \pm 4.81 \\
15.20 \pm 7.26\end{array}$ & -4.20 & $<0.001$ \\
\hline $\begin{array}{l}\text { Cancer stage } \\
\text { III } \\
\text { IV }\end{array}$ & $\begin{array}{r}22(13.9) \\
136(86.1)\end{array}$ & $\begin{array}{l}15.23 \pm 7.62 \\
13.97 \pm 6.88\end{array}$ & 0.78 & 0.435 \\
\hline $\begin{array}{l}\text { Duration after cancer diagnosis (mo) } \\
\leq 12 \\
13-36 \\
\geq 37\end{array}$ & $\begin{array}{c}24.9 \pm 29.6 \\
71(44.9) \\
54(34.2) \\
33(20.9)\end{array}$ & $\begin{array}{l}12.21 \pm 6.50 \\
14.26 \pm 5.39 \\
18.12 \pm 8.57\end{array}$ & 8.92 & $<0.001$ \\
\hline $\begin{array}{l}\text { Surgery for cancer } \\
\text { Yes } \\
\text { No }\end{array}$ & $\begin{array}{r}46(29.1) \\
112(70.9)\end{array}$ & $\begin{array}{l}13.54 \pm 5.59 \\
14.39 \pm 7.48\end{array}$ & 0.78 & 0.436 \\
\hline $\begin{array}{l}\text { Duration after initial chemotherapy (mo) } \\
\leq 12 \\
13-36 \\
\geq 37\end{array}$ & $\begin{array}{c}17.4 \pm 13.9 \\
78(49.4) \\
64(40.5) \\
16(10.1)\end{array}$ & $\begin{array}{l}12.13 \pm 6.25 \\
15.48 \pm 6.16 \\
18.63 \pm 9.94\end{array}$ & 8.48 & $<0.001$ \\
\hline $\begin{array}{l}\text { NCI-CTC grade } \\
\text { I } \\
\text { II } \\
\text { III }\end{array}$ & $\begin{aligned} 50(31.6) \\
102(64.6) \\
6(3.8)\end{aligned}$ & $\begin{array}{l}12.30 \pm 7.58 \\
14.57 \pm 6.42 \\
22.33 \pm 3.67\end{array}$ & 6.11 & 0.003 \\
\hline $\begin{array}{l}\text { Chemotherapeutic agents } \\
\text { Platium } \\
\text { Taxane } \\
\text { Platium+taxane } \\
\text { Others }\end{array}$ & $\begin{array}{l}52(32.9) \\
11(7.0) \\
78(49.4) \\
17(10.7)\end{array}$ & $\begin{array}{l}14.00 \pm 8.04 \\
13.27 \pm 6.34 \\
15.01 \pm 6.68 \\
11.18 \pm 4.16\end{array}$ & 1.51 & 0.214 \\
\hline $\begin{array}{l}\text { Cycles of platinum or taxane chemotherapy } \\
2-5 \\
6-10 \\
11-15 \\
\geq 16\end{array}$ & $\begin{array}{l}9.9 \pm 6.3 \\
38(24.1) \\
45(28.5) \\
50(31.6) \\
25(15.8)\end{array}$ & $\begin{array}{l}13.71 \pm 7.55 \\
13.13 \pm 6.37 \\
14.14 \pm 6.68 \\
16.64 \pm 7.49\end{array}$ & 1.44 & 0.232 \\
\hline
\end{tabular}

Values are presented as mean \pm standard deviation or number $(\%)$.

LUTS, lower urinary tract symptom; ECOG, Eastern Cooperative Oncology Group; NCI-CTC, National Cancer Institute Common Toxicity Criteria. 
pants had gastric cancer, $75.3 \%$ had lung cancer, and $86.1 \%$ had stage IV cancer. The mean length of time after cancer diagnosis was 24.9 months, and $70.9 \%$ of participants did not receive any surgical treatment for cancer. The mean length of time after initial chemotherapy was 17.4 months. Of the participants, $64.6 \%$ were classified as NCI-CTC grade II, and $49.4 \%$ were treated with concomitant platinum and taxane chemotherapy. The mean number of cycles of platinum or taxane chemotherapy was 9.9 (Table 1).

\section{Severity of CIPN and LUTS}

The mean score for CIPN symptoms was 20.17 points. The mean score for LUTS was 14.15 points. The mean score for voiding symptoms was 7.85 points, and the mean score for storage symptoms was 6.37 points. Nocturia (2.67 points) was the most severe symptom, followed by weak urinary flow (2.39

Table 2. Level of neurotoxicity symptoms and lower urinary tract symptoms $(\mathrm{n}=158)$

\begin{tabular}{lc}
\hline Variable (range) & Mean \pm SD \\
\hline Neurotoxicity symptoms (0-44) & $20.17 \pm 10.81$ \\
Sensory (0-4) & $2.07 \pm 1.20$ \\
Hearing (0-4) & $1.32 \pm 1.05$ \\
Motor (0-4) & $2.17 \pm 1.05$ \\
Dysfunction (0-4) & $1.38 \pm 1.23$ \\
IPSS-total (0-41) & $14.15 \pm 6.97$ \\
Voiding symptoms (0-20) & $7.85 \pm 4.49$ \\
Incomplete emptying (0-5) & $1.58 \pm 1.33$ \\
Straining (0-5) & $2.11 \pm 1.40$ \\
Weak stream (0-5) & $2.39 \pm 1.59$ \\
Intermittency (0-5) & $1.80 \pm 1.47$ \\
Storage symptoms (0-15) & $6.37 \pm 3.32$ \\
Frequency (0-5) & $1.89 \pm 1.41$ \\
Urgency (0-5) & $1.80 \pm 1.54$ \\
Nocturia (0-5) & $2.67 \pm 1.21$ \\
IPSS-QoL (0-6) & $3.15 \pm 1.56$
\end{tabular}

SD, standard deviation; IPSS, International Prostate Symptom Score; QoL, Quality of Life.

Table 3. Severity of lower urinary tract symptoms $(n=158)$

\begin{tabular}{lr}
\hline IPSS severity (score) & No. (\%) \\
\hline Mild (0-7) & $26(16.5)$ \\
Moderate (8-19) & $100(63.3)$ \\
Severe (20-35) & $32(20.2)$ \\
\hline
\end{tabular}

IPSS, International Prostate Symptom Score. points) and straining (2.11 points). The average IPSS-QoL score was 3.15 points (Table 2). Twenty-five participants (16.5\%) had mild LUTS, 100 (63.3\%) had moderate LUTS, and 32 (20.2\%) had severe LUTS (Table 3).

\section{Relationship Between LUTS and CIPN Symptoms}

There was a moderate positive relationship between CIPN symptoms and LUTS $(\mathrm{r}=0.53, \mathrm{P}<0.001)$. In other words, severe CIPN symptoms were associated with severe LUTS (Fig. 1).

\section{Differences in LUTS According to the Participants' General Characteristics}

Differences were found in LUTS according to age, ECOG score, diagnosis, the length of time after cancer diagnosis, the length of time after initial chemotherapy, and NCI-CTC grade (Table 1).

\section{Factors Influencing LUTS}

This study investigated various factors that were related with LUTS, such as general characteristics, ECOG score, diagnosis, the length of time after cancer diagnosis, the length of time after initial chemotherapy, the length of time after the onset of CIPN symptoms, and the severity of CIPN symptoms. All these factors were analyzed as independent variables, and there was no issue of multicollinearity among these variables.

The regression model for LUTS showed a significant relationship $(\mathrm{F}=10.20, \mathrm{P}<0.001)$, with an explanatory power of $40 \%$. The length of time after cancer diagnosis and the severity of CIPN symptoms were the main factors that significantly influenced LUTS. The severity of CIPN symptoms was the most

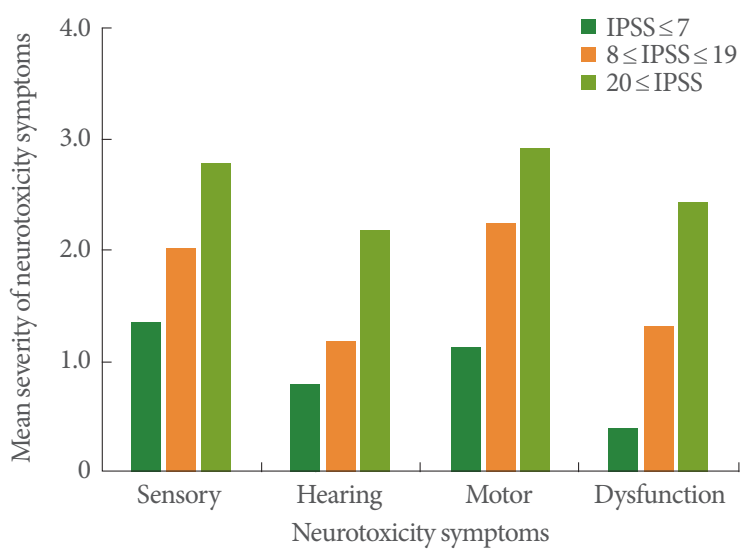

Fig. 1. Association of lower urinary tract symptom severity and neurotoxicity symptoms. IPSS, International Prostate Symptom Score. 
Table 4. Factors influencing lower urinary tract symptoms ${ }^{\mathrm{a}}(\mathrm{n}=158)$

\begin{tabular}{lccccc}
\hline Predictor & $\mathrm{B}$ & $\mathrm{SE}$ & Standardized $\beta$ & $\mathrm{t}$ & $\mathrm{P}$-value \\
\hline Intercept & -3.18 & 3.10 & 0.00 & 0.306 & 0.03 \\
ECOG (reference: 1) & 0.05 & 0.80 & 0.01 & 0.06 & 1.44 \\
Diagnosis (reference: stomach cancer) & 1.56 & 1.08 & 0.10 & 0.154 \\
Duration after cancer diagnosis (mo) & 2.52 & 1.23 & 0.15 & 0.05 & 0.79 \\
Duration after initial chemotherapy (mo) & 0.95 & 1.22 & 0.07 & 0.434 \\
Neuropathy symptoms (FACT-GOG/NTx score) & 0.27 & 0.05 & 0.43 & 5.33 & $<0.001$ \\
\hline
\end{tabular}

SE, standard error; ECOG, Eastern Cooperative Oncology Group; FACT-GOG/NTx, Functional Assessment of Cancer therapy/Gynecology Oncology Group/Neurotoxicity.

$\mathrm{R}^{2}=0.40$, adjusted $\mathrm{R}^{2}=0.36, \mathrm{~F}(\mathrm{P}$-value $)=10.20(<0.001)$.

${ }^{a}$ Adjusted for age.

influential factor (Table 4).

\section{DISCUSSION}

The present study investigated LUTS and factors influencing LUTS in advanced cancer patients with CIPN symptoms. The mean LUTS score was 14.15 points (out of 35 points), indicating moderate severity. This score was higher than the LUTS score in patients with invasive bladder cancer (between 6.6 and 7.1 points) reported in a previous study [8] and the LUTS score for rectal cancer patients receiving chemotherapy (6.2 points) reported by Kim et al. [17].

In this study, $16.5 \%$ of advanced cancer patients with CIPN had mild LUTS, $63.3 \%$ had moderate LUTS, and $20.2 \%$ had severe LUTS. This finding shows a trend for a higher proportion of moderate and severe LUTS than was found in advanced prostate cancer patients receiving brachytherapy (mild, 50.0\%; moderate, 39.0\%; and severe, 6.0\%) [18]. In this study, the IPSSQoL score and the micturition-associated QoL score were 3.15 of 6 possible points, which was higher than the score for IPSSQoL (2.7 points) in rectal cancer patients following rectal resection with autonomic nerve preservation [17]. LUTS have been found to negatively influence the QoL of cancer patients [8].

In the present study, LUTS severity was positively related to CIPN symptoms. No previous studies have investigated the relationship between LUTS and CIPN symptoms; therefore, it was not possible to compare the results of this study to those of other previous studies. However, the study of cervical cancer patients by Benedetti-Panici [19] reported that LUTS such as sensory loss, difficult micturition, and severe urinary incontinence occurred in $26 \%$ of cervical cancer patients after 12 months of treatment with platinum anticancer drugs. Stubble- field et al. [5] considered that areflexia, LUTS such as urinary retention, and CIPN symptoms might occur after the administration of anticancer drugs such as platinum and paclitaxel, indirectly suggesting that CIPN symptoms may be relevant to LUTS. However, the present study was conducted using a subjective self-administered questionnaire; therefore, it had a limited capability to identify objective causality. Further studies using objective measurements are needed to clarify the causality.

In the present study, LUTS increased in severity with age, and it can be assumed that the aging process of the body may decrease bladder muscle tension and increase bladder volume or the sensation of residual urine, resulting in more severe LUTS; thus, the prevalence of storage LUTS and voiding LUTS such as frequency and nocturia increases with age [20,21]. In addition, older patients may have an increased incidence of adverse effects associated with chemotherapy, as well as a decreased ability to cope with them [22]. Therefore, attention should be paid not only to the severity of LUTS, but also to the cause and subsequent effects of LUTS in elderly cancer patients. In particular, the relationships of older age and male sex with the severity of LUTS should be analyzed.

Even though the present study found no sex-related differences in LUTS, previous reports have found that males had more severe LUTS than females $[23,24]$; further studies of the effect of sex on the relationship between CIPN symptoms and LUTS severity are therefore warranted.

A higher ECOG score in advanced cancer patients was associated with more severe LUTS. Gift et al. [9] reported that because cancer patients perceived greater weakness, fatigue, and physical limitations than other patients, they complained more of nocturia symptoms. A decrease in physical activity was reported to be a risk factor for the onset of LUTS [25]. Study par- 
ticipants with NCI-CTC grade II/III CIPN symptoms were found to have severe LUTS. Individuals with an NCI-CTC grade II/III have impaired daily life activities or severe functional impairment due to sensory loss or paresthesia [26]. It can be assumed that, in NCI-CTC grade II/III patients, the bladder becomes rigid or contracted, thereby leading to the development of symptoms such as nocturia and straining while voiding.

In the present study, longer lengths of time following cancer diagnosis and initial chemotherapy were associated with more severe LUTS. This tendency likely occurred because advanced cancer patients had been repeatedly administered neurotoxic anticancer agents such as taxane or platinum compounds for the treatment of invasive sites. The severity of CIPN symptoms increased as the number of administered doses increased [5,26]. Long-term administration of oxaliplatin or cisplatin might lead to sympathetic and parasympathetic nervous system dysfunction, and has been found to result in urogenital dysfunction [27]. Additionally, a previous study reported that the cumulative dose of paclitaxel or docetaxel was closely clinically related with the degree of chemotherapy-related peripheral neurotoxicity, and that cumulative doses in line with treatment progression were accompanied by motor or autonomic impairment [28]. However, the length of time after the last chemotherapy session and the number of chemotherapy cycles were not investigated in this study. Cumulative or nerve damage and a short interval after chemotherapy might aggravate voiding dysfunction. Further studies are required to determine the effects of the cumulative dose of anticancer agents on LUTS.

Multiple regression analysis was performed to investigate the factors influencing LUTS in advanced cancer patients with CIPN symptoms. The length of time following cancer diagnosis and the severity of CIPN symptoms were found to be key factors influencing LUTS, and the severity of CIPN symptoms showed the greatest influence. Windebank and Grisold [27] stated that the length of chemotherapy treatment and preexisting neuropathy influenced the development and the severity of CIPN, and autonomic dysfunction or urogenital dysfunction of the urinary bladder occurred in patients receiving long-term administration of neurotoxic anticancer agents such as oxaliplatin or vincristine. Cancer patients with LUTS may have a reduced QoL because they are more restricted in their physical functions and social roles than those without LUTS [8]. Therefore, continuous assessment and management of LUTS, as well as CIPN symptoms, are important for advanced cancer patients even after treatment.
This study investigated LUTS in advanced gastric and lung cancer patients with concurrent CIPN symptoms; therefore, these results cannot be generalized to all cancer patients. As such, further studies are required to expand the scope of participants to include advanced solid cancer patients with CIPN symptoms, such as patients with metastatic colon, breast, or ovarian cancer [4] and to investigate the relationship between CIPN symptoms and LUTS. In addition, studies should consider not only the length of time after cancer diagnosis, but also the duration of the cancer stage related to LUTS.

This study had a limitation in that it did not identify alphablockers or anticholinergics, which may influence bladder function, among the drugs administered to cancer patients. This study included a relatively large sample of male patients who were older than 60 , and therefore could have been vulnerable to selection bias. This was a cross-sectional study; thus, it would be difficult to compare IPSS scores before and after chemotherapy.

Another limitation of this study is that it did not extensively consider the various comorbid conditions that can affect LUTS. Such conditions include arthritis, asthma, depression, diabetes, heart disease, neurological conditions, and recurrent urinary tract infections [29]. Further studies are needed of the effect of general status or performance in addition to the ECOG score. The prevalence of LUTS may increase in patients with symptoms of depression [23]; therefore, studies on the effects of LUTS on comorbid conditions and psychological variables may be worthwhile.

In conclusion, nocturia was found to be the most prevalent LUTS in advanced cancer patients experiencing CIPN. The length of time after cancer diagnosis and the severity of CIPN were found to be factors influencing LUTS. In particular, the severity of CIPN symptoms was the most important predictor of LUTS.

Therefore, patients' urination status should be considered before initiating treatment with anticancer drugs that can cause CIPN. We suggest that nurses caring for advanced cancer patients should conduct a comprehensive health assessment that includes a history of treatment, physical neuropathic symptoms and severity, mobility, daily life activities, and LUTS, for any patient complaining of CIPN symptoms. In practice, it is necessary to develop micturition-related nursing assessment records and nursing intervention algorithms, and to provide CIPN information and education regarding LUTS management to the relevant patients prior to initiation of chemotherapy. 


\section{AUTHOR CONTRIBUTION STATEMENT}

- Full access to all the data in the study and takes responsibility for the integrity of the data and the accuracy of the data analysis: $\mathrm{OHC}$

- Study concept and design: $\mathrm{OHC}$

- Acquisition of data: RHP

- Analysis and interpretation of data: $\mathrm{OHC}$

- Drafting of the manuscript: $\mathrm{KHH}$

- Critical revision of the manuscript for important intellectual content: $\mathrm{KHH}$

- Statistical analysis: $\mathrm{OHC}$

- Administrative, technical, or material support: JCK

-Study supervision: YSY

\section{REFERENCES}

1. Camps C, Caballero C, Blasco A, Safont MJ, Berrocal A, Garde J, et al. Weekly paclitaxel as second/third-line treatment in advanced non-small cell lung cancer patients: efficacy and tolerability. Anticancer Res 2005;25(6C):4611-4.

2. Kautio AL, Haanpää M, Kautiainen H, Kalso E, Saarto T. Burden of chemotherapy-induced neuropathy--a cross-sectional study. Support Care Cancer 2011;19:1991-6.

3. Yoo YS, Cho OH. Relationship between quality of life and nurseled bedside symptom evaluations in patients with chemotherapyinduced peripheral neuropathy. Asian Nurs Res (Korean Soc Nurs Sci) 2014;8:36-41.

4. Grisold W, Cavaletti G, Windebank AJ. Peripheral neuropathies from chemotherapeutics and targeted agents: diagnosis, treatment, and prevention. Neuro Oncol 2012;14 Suppl 4:iv45-54.

5. Stubblefield MD, Burstein HJ, Burton AW, Custodio CM, Deng GE, Ho M, et al. NCCN task force report: management of neuropathy in cancer. J Natl Compr Canc Netw 2009;7 Suppl 5:S1-S26

6. Abrams P, Cardozo L, Fall M, Griffiths D, Rosier P, Ulmsten U, et al. The standardisation of terminology of lower urinary tract function: report from the Standardisation Sub-committee of the International Continence Society. Neurourol Urodyn 2002;21:167-78.

7. Faithfull S, Lemanska A, Aslet P, Bhatt N, Coe J, Drudge-Coates L, et al. Integrative review on the non-invasive management of lower urinary tract symptoms in men following treatments for pelvic malignancies. Int J Clin Pract 2015;69:1184-208.

8. Hsieh CI, Lung AL, Chang LI, Sampselle CM, Lin CC, Liao YM. Prevalence, associated factors, and relationship to quality of life of lower urinary tract symptoms: a cross-sectional, questionnaire sur- vey of cancer patients. Int J Clin Pract 2013;67:566-75.

9. Gift AG, Jablonski A, Stommel M, Given CW. Symptom clusters in elderly patients with lung cancer. Oncol Nurs Forum 2004;31:20212.

10. White AJ, Reeve BB, Chen RC, Stover AM, Irwin DE. Urinary incontinence and health-related quality of life among older Americans with and without cancer: a cross-sectional study. BMC Cancer 2013;13:377.

11. Marinkovic SP, Rovner ES, Moldwin RM, Stanton SL, Gillen LM, Marinkovic CM. The management of overactive bladder syndrome. BMJ 2012;344:e2365.

12. Payne H, Adamson A, Bahl A, Borwell J, Dodds D, Heath C, et al. Chemical- and radiation-induced haemorrhagic cystitis: current treatments and challenges. BJU Int 2013;112:885-97.

13. Lange MM, Maas CP, Marijnen CA, Wiggers T, Rutten HJ, Kranenbarg EK, et al. Urinary dysfunction after rectal cancer treatment is mainly caused by surgery. Br J Surg 2008;95:1020-8.

14. Calhoun EA, Welshman EE, Chang CH, Lurain JR, Fishman DA, Hunt TL, et al. Psychometric evaluation of the Functional Assessment of Cancer Therapy/Gynecologic Oncology Group-Neurotoxicity (Fact/GOG-Ntx) questionnaire for patients receiving systemic chemotherapy. Int J Gynecol Cancer 2003;13:741-8.

15. Barry MJ, Fowler FJ Jr, O'Leary MP, Bruskewitz RC, Holtgrewe HL, Mebust WK, et al. The American Urological Association symptom index for benign prostatic hyperplasia. The Measurement Committee of the American Urological Association. J Urol 1992;148: 1549-57.

16. Choi HR, Chung WS, Shim BS, Kwon SW, Hong SJ, Chung BH, et al. Translation validity and reliability of I-PSS Korean Version. Korean J Urol 1996;37:659-65.

17. Kim NK, Aahn TW, Park JK, Lee KY, Lee WH, Sohn SK, et al. Assessment of sexual and voiding function after total mesorectal excision with pelvic autonomic nerve preservation in males with rectal cancer. Dis Colon Rectum 2002;45:1178-85.

18. Hoskin P, Rojas A, Ostler P, Hughes R, Alonzi R, Lowe G, et al. High-dose-rate brachytherapy alone given as two or one fraction to patients for locally advanced prostate cancer: acute toxicity. Radiother Oncol 2014;110:268-71.

19. Benedetti-Panici P, Zullo MA, Plotti F, Manci N, Muzii L, Angioli R. Long-term bladder function in patients with locally advanced cervical carcinoma treated with neoadjuvant chemotherapy and type 3-4 radical hysterectomy. Cancer 2004;100:2110-7.

20. Chung HS, Kim JS, Kim SO, Kim HS, Kwon D, Park K, et al. Age related changes of voiding patterns in women with overactive bladder. J Korean Continence Soc 2009;13:37-44. 
21. Lee YS, Lee KS, Jung JH, Han DH, Oh SJ, Seo JT, et al. Prevalence of overactive bladder, urinary incontinence, and lower urinary tract symptoms: results of Korean EPIC study. World J Urol 2011; 29:185-90.

22. Wickham R. Chemotherapy-induced peripheral neuropathy: a review and implications for oncology nursing practice. Clin J Oncol Nurs 2007;11:361-76.

23. Choi SH, Kim ST, Park HM, Moon KH, Jung JH, Cha HE. Analysis of characteristics and steroid effects in olfactory dysfunction patients. J Rhinol 2016;23:39-43.

24. Kupelian V, Wei JT, O’Leary MP, Kusek JW, Litman HJ, Link CL, et al. Prevalence of lower urinary tract symptoms and effect on quality of life in a racially and ethnically diverse random sample: the Boston Area Community Health (BACH) Survey. Arch Intern Med 2006;166:2381-7.
25. Litman HJ, Steers WD, Wei JT, Kupelian V, Link CL, McKinlay JB. Relationship of lifestyle and clinical factors to lower urinary tract symptoms: results from Boston Area Community Health survey. Urology 2007;70:916-21.

26. Argyriou AA, Polychronopoulos P, Iconomou G, Chroni E, Kalofonos HP. A review on oxaliplatin-induced peripheral nerve damage. Cancer Treat Rev 2008;34:368-77.

27. Windebank AJ, Grisold W. Chemotherapy-induced neuropathy. J Peripher Nerv Syst 2008;13:27-46.

28. Cavaletti G, Alberti P, Frigeni B, Piatti M, Susani E. Chemotherapyinduced neuropathy. Curr Treat Options Neurol 2011;13:180-90.

29. Coyne KS, Kaplan SA, Chapple CR, Sexton CC, Kopp ZS, Bush EN, et al. Risk factors and comorbid conditions associated with lower urinary tract symptoms: EpiLUTS. BJU Int 2009;103 Suppl 3:24-32. 